\title{
Genome Sequencing of Streptomyces olivaceus SCSIO T05 and Activated Production of Lobophorin CR4 via Metabolic Engineering and Genome Mining
}

\author{
Chunyan Zhang ${ }^{1,2}$, Wenjuan Ding ${ }^{1,2}$, Xiangjing Qin ${ }^{1}$ and Jianhua Ju ${ }^{1,2, *(\mathbb{D})}$ \\ 1 CAS Key Laboratory of Tropical Marine Bio-resources and Ecology, Guangdong Key Laboratory of Marine \\ Materia Medica, RNAM Center for Marine Microbiology, South China Sea Institute of Oceanology, \\ Chinese Academy of Sciences, 164 West Xingang Road, Guangzhou 510301, China; \\ zhchuny@foxmail.com (C.Z.); 13760785354@163.com (W.D.); xj2005qin@126.com (X.Q.) \\ 2 College of Oceanology, University of Chinese Academy of Sciences, 19 Yuquan Road, Beijing 100049, China \\ * Correspondence: jju@scsio.ac.cn; Tel./Fax: +86-20-8902-3028
}

Received: 18 September 2019; Accepted: 16 October 2019; Published: 20 October 2019

\begin{abstract}
Marine-sourced actinomycete genus Streptomyces continues to be an important source of new natural products. Here we report the complete genome sequence of deep-sea-derived Streptomyces olivaceus SCSIO T05, harboring 37 putative biosynthetic gene clusters (BGCs). A cryptic BGC for type I polyketides was activated by metabolic engineering methods, enabling the discovery of a known compound, lobophorin CR4 (1). Genome mining yielded a putative lobophorin BGC (lbp) that missed the functional FAD-dependent oxidoreductase to generate the D-kijanose, leading to the production of lobophorin CR4 without the attachment of D-kijanose to C17-OH. Using the gene-disruption method, we confirmed that the $l b p$ BGC accounts for lobophorin biosynthesis. We conclude that metabolic engineering and genome mining provide an effective approach to activate cryptic BGCs.
\end{abstract}

Keywords: genome sequencing; gene disruption; lobophorin; metabolic engineering; genome mining

\section{Introduction}

Microbially produced natural products (NPs) are an important reservoir of therapeutic and agricultural agents [1]. In the previous years, quantities of new bioactive NPs were isolated from marine-derived Streptomyces strains, suggesting marine-derived Streptomyces as a predominant source of new NPs [2]. In recent years, whole-genome sequencing programs have made it clear that microorganisms have greater biosynthetic potential but are mostly underexplored by virtue that most biosynthetic gene clusters (BGCs) in a single microbial genome are normally silent. Activation of these silent BGCs contributes to new NPs discoveries. Zhang and co-workers activated a cryptic polycyclic tetramate macrolactam (PTM) BGC in Streptomyces pactum SCSIO 02999 by promoter engineering and heterologous expression [3], and also promoted the expression of a silent PKS/NRPS hybrid BGC in the same Streptomyces strain by the alteration of several regulatory genes [4]. The production of nocardamine [5] and atratumycin [6] in Streptomyces atratus SCSIO ZH16 was turned on via metabolic engineering. These genome-based studies exemplify the benefits of genome mining and metabolic engineering used for activating cryptic BGCs and discovering new bioactive NPs.

Lobophorins (Supporting Information (SI), Figure S1) belonging to a large class of spirotetronate antibiotics structurally feature a tetronate moiety spiro-linked with a cyclohexene ring, which is called pentacyclic aglycon or kijanolide [7-17]. Almost all of this class of compounds has a broad spectrum of antibacterial activities, as well as antitumor activity. Efforts to produce more spirotetronate antibiotics for drug discovery have thrived. Owing to the structural complexity of this family member, biosynthesis seems to be an effective way to afford the production of spirotetronate antibiotics, providing access to 
new analogues by pathway engineering and combinatorial biosynthetic approaches. In this paper, we report (i) the complete genome sequence of a deep-sea-derived Streptomyces olivaceus SCSIO T05, a talented strain capable of producing an array of putative NPs; (ii) activation of a cryptic lobophorin BGC (lbp) by mutagenetic methods and isolation of one known spirotetronate antibiotic lobophorin CR4 (1); and (iii) identification of the lbp BGC housed in S. olivaceus SCSIO T05 by gene-disruption experiment and bioinformatics analysis.

\section{Results and Discussion}

\subsection{Genome Sequencing and Annotation of Streptomyces olivaceus SCSIO T05}

Whole genome sequence is important when analyzing the potential production of secondary metabolites [5,18]. S. olivaceus SCSIO T05, a marine-derived strain, was previously reported to be isolated from the Indian Ocean deep-sea-derived sediment [19]. Its draft genome sequence was first gained by Illumina sequencing technology, but with several gap regions. In order to estimate the biosynthetic potential of S. olivaceus SCSIO T05, the complete genome was re-sequenced and acquired by the single-molecule real-time (SMRT) sequencing technology (PacBio). A total of 67156 filtered reads with high-quality data of $432570025 \mathrm{bp}$ were generated, and then they were assembled into a linear contig by the hierarchical genome assembly process (HGAP) [20]. The complete genome revealed that 8458055 base pairs constitute a linear chromosome without a plasmid, with $72.51 \%$ of GC content (Figure 1 and Table 1). Totally, 7700 protein-coding genes were predicted, along with 18 rRNA and 65 tRNA. The genome sequence of S. olivaceus SCSIO T05 was deposited in GenBank (CP043317).

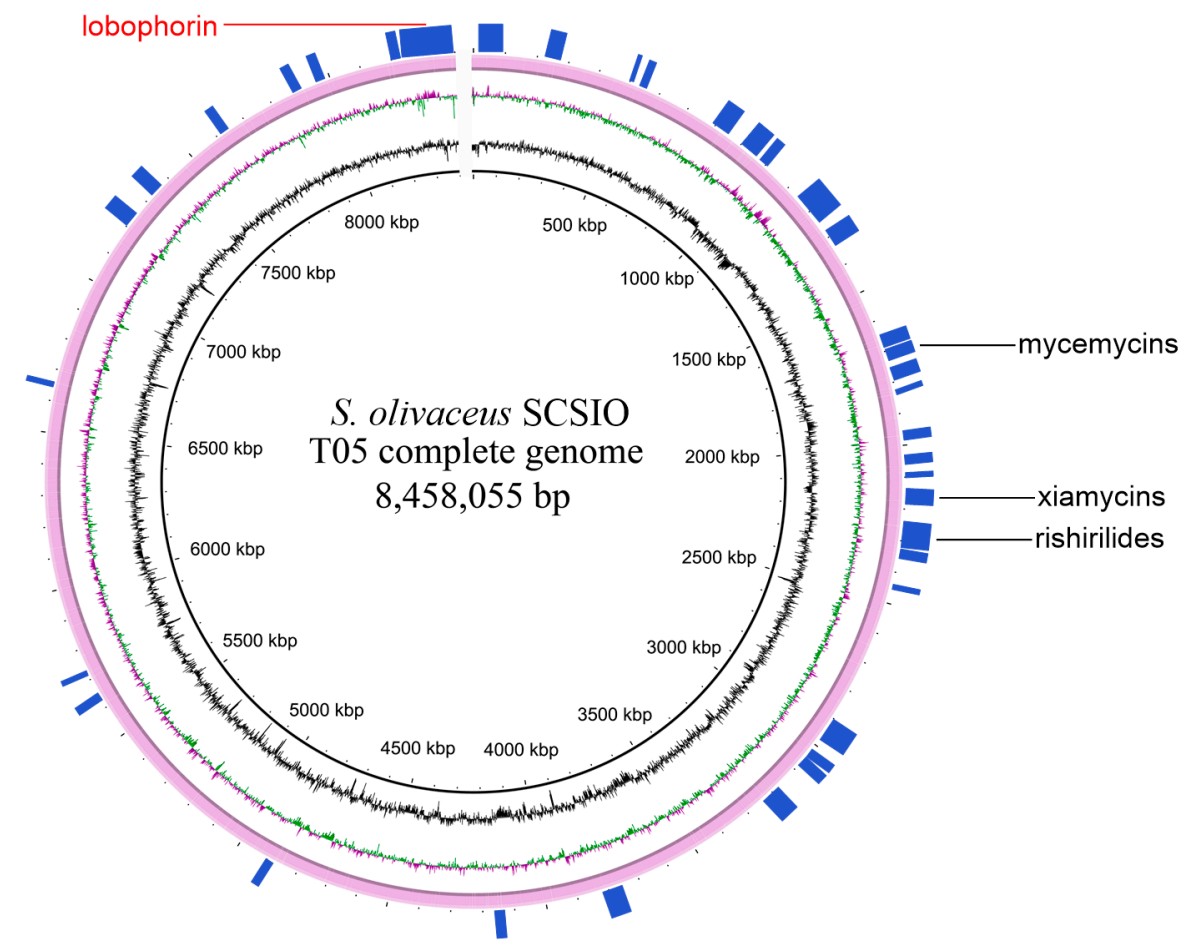

Figure 1. The complete genome of S. olivaceus SCSIO T05. The three circles (inner to outer) represent forward GC content, GC skew, and the distribution of putative biosynthetic gene clusters (BGCs) (represented by the bars) generated by antiSMASH 5.0. Clusters 18, 17, and 11 were described as rishirilides, xiamycins, and mycemycins BGCs, respectively. The putative lobophorin BGC with red color was referred to as cluster 37. 
Table 1. Genome features of S. olivaceus SCSIO T05.

\begin{tabular}{cc}
\hline Feature & Value \\
\hline Genome size $(\mathrm{bp})$ & $8,458,055$ \\
Average GC content $(\%)$ & 72.51 \\
Protein-coding genes & 7700 \\
Total size of Protein-coding genes $(\mathrm{bp})$ & $7,543,173$ \\
rRNAs number & 18 \\
tRNAs number & 65 \\
\hline
\end{tabular}

AntiSMASH analysis by using antiSMASH 5.0 [21] suggested 37 BGCs within the S. olivaceus SCSIO T05 genome (Figure 1 and Table 2). The 37 BGCs totally occupy $1.59 \mathrm{Mb}, 18.76 \%$ of the complete genome. Most of the BGCs distribute in the two subtelomeric regions of the genome of some Streptomyces strains [18] and so do the BGCs in S. olivaceus SCSIO T05 genome. It is predicted that several BGCs are responsible for the production of polyketide- and nonribosome-peptide-derived secondary metabolites, including four PKS (Type I, Type II and Type III) and six NRPS, and six hybrid BGCs possess genes encoding more than one type of scaffold-synthesizing enzyme. Twenty-one BGCs are predicted to produce terpene, bacteriocin, lanthipeptide, or other categories. This analysis indicates that S. olivaceus SCSIO T05 is capable of producing an array of secondary metabolites, serving as a target strain for further metabolic engineering and genome mining.

Table 2. AntiSMASH-predicted BGCs for S. olivaceus SCSIO T05.

\begin{tabular}{|c|c|c|c|}
\hline \multirow{2}{*}{ BGC } & \multicolumn{2}{|c|}{ Position } & \multirow{2}{*}{ Type (Product) } \\
\hline & From & To & \\
\hline Cluster 1 & 2725 & 89768 & Type I Polyketide synthase (T1 PKS) \\
\hline Cluster 2 & 234616 & 284137 & Non-ribosomal peptide synthetase (NRPS) cluster \\
\hline Cluster 3 & 504553 & 512728 & $\begin{array}{c}\text { Bacteriocin or other unspecified ribosomally synthesized and } \\
\text { post-translationally modified peptide product (RiPP) } \\
\text { cluster (Bacteriocin) }\end{array}$ \\
\hline Cluster 4 & 525945 & 544617 & Terpene \\
\hline Cluster 5 & 793277 & 855894 & NRPS \\
\hline Cluster 6 & 901333 & 979368 & T1 PKS \\
\hline Cluster 7 & 980891 & 1005613 & Lanthipeptide cluster (Lanthipeptide) \\
\hline Cluster 8 & 1135886 & 1240760 & Other types of PKS cluster (Otherks)-NRPS \\
\hline Cluster 9 & 1275164 & 1347740 & NRPS-Terpene \\
\hline Cluster 10 & 1651711 & 1694648 & NRPS-Nucleoside cluster (Nucleoside) \\
\hline Cluster 11 & 1695020 & 1734380 & Otherks \\
\hline Cluster 12 & 1751698 & 1796277 & NRPS \\
\hline Cluster 13 & 1840051 & 1851963 & Siderophore cluster (Siderophore) \\
\hline Cluster 14 & 1967451 & 1990613 & Lanthipeptide \\
\hline Cluster 15 & 2037772 & 2059400 & Terpene \\
\hline Cluster 16 & 2090680 & 2102023 & Bacteriocin \\
\hline Cluster 17 & 2138860 & 2187226 & T1PKS-NRPS \\
\hline Cluster 18 & 2230691 & 2317060 & NRPS-Type II PKS (T2 PKS)-Otherks \\
\hline Cluster 19 & 2330735 & 2352337 & Lanthipeptide \\
\hline Cluster 20 & 2443907 & 2456009 & Siderophore \\
\hline Cluster 21 & 2905748 & 2978302 & T2 PKS \\
\hline Cluster 22 & 3029068 & 3048760 & Terpene \\
\hline Cluster 23 & 3049806 & 3075321 & Beta-lactone containing protease inhibitor (Betalactone) \\
\hline Cluster 24 & 3182776 & 3235915 & NRPS \\
\hline Cluster 25 & 3764472 & 3822515 & NRPS \\
\hline Cluster 26 & 4131410 & 4159582 & Lanthipeptide \\
\hline Cluster 27 & 4881296 & 4901736 & Phenazine cluster (Phenazine) \\
\hline
\end{tabular}


Table 2. Cont.

\begin{tabular}{cccc}
\hline \multirow{2}{*}{ BGC } & \multicolumn{2}{c}{ Position } & Type (Product) \\
\cline { 2 - 3 } & From & To & \\
\hline Cluster 28 & 5633979 & 5656500 & Lasso peptide cluster (Lassopeptide) \\
Cluster 29 & 5716930 & 5727556 & Melanin cluster (Melanin) \\
Cluster 30 & 6667385 & 6677783 & Ectoine cluster (Ectoine) \\
Cluster 31 & 7200930 & 7253804 & NRPS \\
Cluster 32 & 7328818 & 7368924 & Type III PKS (T3 PKS) \\
Cluster 33 & 7614814 & 7636052 & Aminoglycoside/aminocyclitol cluster (Amglyccycl) \\
Cluster 34 & 7882883 & 7906528 & Terpene \\
Cluster 35 & 7959695 & 7980831 & Indole cluster (Indole) \\
Cluster 36 & 8200560 & 8221618 & Terpene \\
Cluster 37 & 8239655 & 8455702 & T1pks-Nrps-T3 PKS-Oligosaccharide cluster (Oligosaccharide)-Other \\
\hline
\end{tabular}

\subsection{Activation of a Cryptic Lobophorin BGC in the Genetically Engineered Mutant}

In actuality, only a minority of potential chemicals are produced under standard laboratory culture conditions. Furthermore, the corresponding products are likely to be overlooked for multiple reasons, including low production rates, a large metabolic background, or improper culture conditions [22]. Fermented using modified-RA medium, the secondary metabolites produced by S. olivaceus SCSIO T05 were subsequently profiled using HPLC-DAD-UV. Multiple peaks were detected in the fermentation extract (Figure 2, trace i). We previously reported that five known NPs, rishirilides B (2) and C (3), lupinacidin A (4), galvaquinone B (5), and xiamycin A (6), were produced as major secondary metabolites from the wild-type strain $[19,23]$. In addition, an orphan dibenzoxazepinone biosynthetic pathway was mutagenically activated, leading to the production of new mycemycins [24], suggesting that S. olivaceus SCSIO T05 has a great potential for producing new NPs.

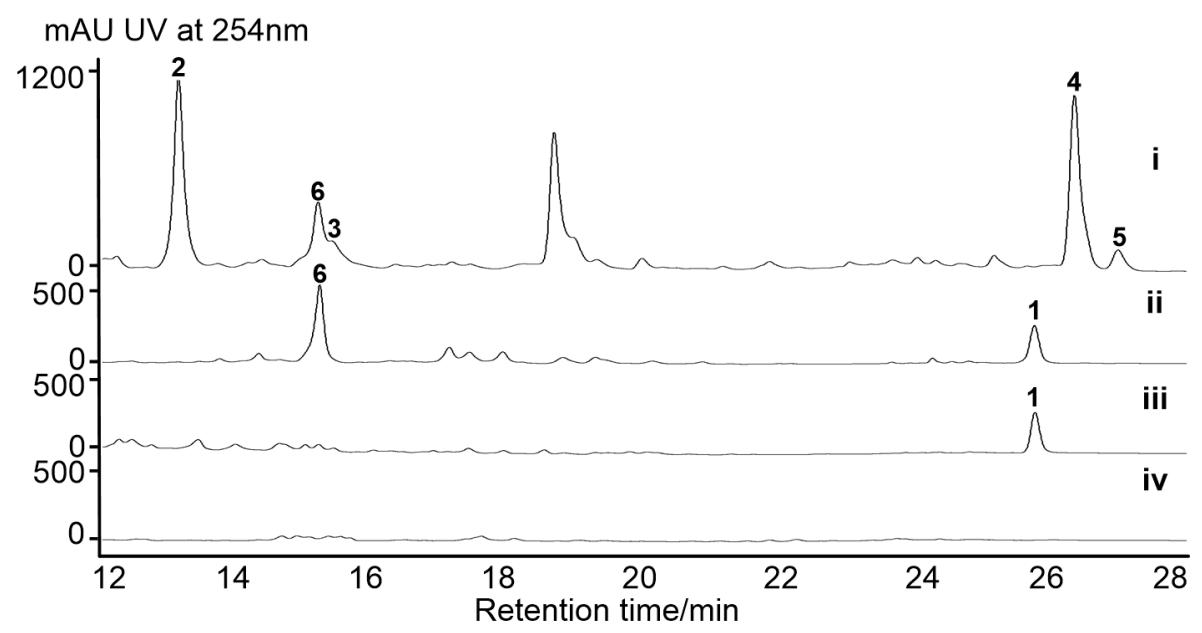

Figure 2. HPLC-based analyses of fermentation broths: (i) S. olivaceus SCSIO T05; (ii) S. olivaceus SCSIO T05R; (iii) S. olivaceus SCSIO T05RX; and (iv) S. olivaceus SCSIO T05RXL. Compound $\mathbf{1}$ is lobophorin CR4. Compounds 2-6 were previously identified as rishirilide $B$, rishirilide $C$, lupinacidin A, galvaquinone $\mathrm{B}$, and xiamycin A, respectively.

For exploring other secondary metabolites from the strain, S. olivaceus SCSIO T05/ $\Delta r s d K_{2}(S$. olivaceus SCSIO T05R) was constructed to abolish the production of the anthracenes [19]. The production of the second major secondary metabolites xiamycins was accumulated, along with a new peak around $26 \mathrm{~min}$, distinct from the UV absorption characteristics of xiamycins (Figure 2, trace ii). For further background elimination of xiamycins, a "double-deletion" mutant $S$. olivaceus SCSIO T05/ $\Delta r s d K_{2} / \Delta x m c P$ (S. olivaceus SCSIO T05RX) was constructed [23] in which the new peak (1) appeared to be the major product (Figure 2, trace iii). Accordingly, the S. olivaceus SCSIO T05RX mutant was fermented at a large scale, enabling the isolation and structure elucidation of this newly generated compound. 
It was identified as a known compound designated as lobophorin CR4 (Figure 3), by comparing HRESIMS, ${ }^{1} \mathrm{H}$, and ${ }^{13} \mathrm{C}$ NMR data (SI, Figures S2-S4) to the reported data of an intermediate isolated from the Streptomyces sp. SCSIO 01127/DlobG1 mutant [11]. It is reported that shifting metabolic flux of a wild-type strain by blocking the predominant product pathways may afford new secondary metabolites [5]. During our efforts to acquire new secondary metabolites by shifting the metabolic flux of marine actinomycetes $[5,23,24]$, the production of nocardamine, olimycins, and mycemycins was turned on at the expense of major products by using gene knock-out methods. Similarly, the "double-deletion" mutant (S. olivaceus SCSIO T05RX) was constructed to abolish the production of two major secondary metabolites, anthracenes and xiamycins, from the wild-type strain [19,23]. With the engineered shifting of S. olivaceus metabolic flux, the newly produced lobophorin CR4 was activated.

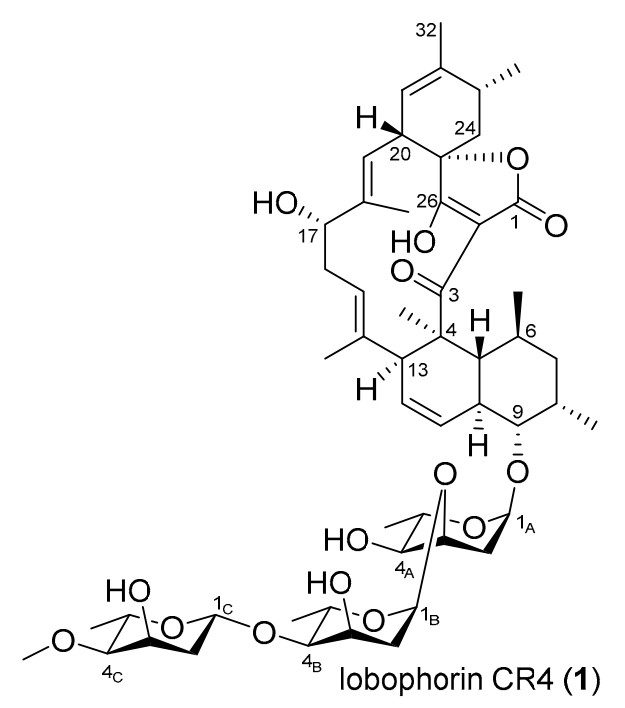

Figure 3. Structure of the isolated lobophorin CR4.

\subsection{Identification of a Putative Lobophorin (lbp) BGC via Genome Mining}

The antiSMASH analysis of the complete genome of S. olivaceus SCSIO T05 revealed a $99.1 \mathrm{~kb}$ type I PKS BGC named as lobophorin BGC $(l b p)$, showing highly similar traits to the reported lob BGCs from Streptomyces sp. FXJ7.023 [16] and Streptomyces sp. SCSIO 01127 [11]. The complete lbp contains 38 open reading frames (ORFs). The genetic organization of $l b p$ is shown in Figure $4 \mathrm{~A}$, with genes color-coded on the basis of their proposed functions summarized in Table 3 . The nucleotide sequences were deposited in GenBank (MN396889). The lbp BGC contains six inconsecutive genes $l b p A 1-A 6$, similar to $l o b A 1-A 5$ in $l o b$ from $S$. sp. SCSIO 01127. Differently, the LobA4 homologue is separated into two polyketide synthases (PKSs), LbpA4 and LbpA5, in lbp. The high similarity between the PKS modules in $l b p$ and in $l o b$ enables us to propose that the assembly of the linear polyketide chain catalyzed by LbpA1-A6 utilizes six malonyl CoAs, six methylmalonyl-CoAs, and a 3-carbon glycerol unit (Figure 5) [11]. The lbp harbors four putative regulator genes (lbpR1-R4) (Figure 4 and Table 3) that are highly similar to $l o b R 1, l o b R 3, l o b R 4$, and $l o b R 5$ in $l o b$, respectively. These four regulators are assumed to be involved in the regulation network of lobophorin CR4 biosynthesis, which seems to be less complex than lob but more complex than $k i j$ [7] and $t c a$ [8]. In contrast, five regulator genes lobR1-R5 are identified in lob; three regulator genes, kijA8, kijC5, and kijD12, are included in kij; tcaR1 and tcaR2 both encode regulators in $t c a$. There is only one gene, $l b p U 2$ in $l b p$, with no apparent homologue in $l o b$ (Figure 4 and Table 3). The other genes included in $l b p$ are putatively associated with the biosynthesis of kijanose and L-digitoxose units by virtue of high similarities to corresponding counterparts in $l o b$ (Figure 4 and Table 3). 

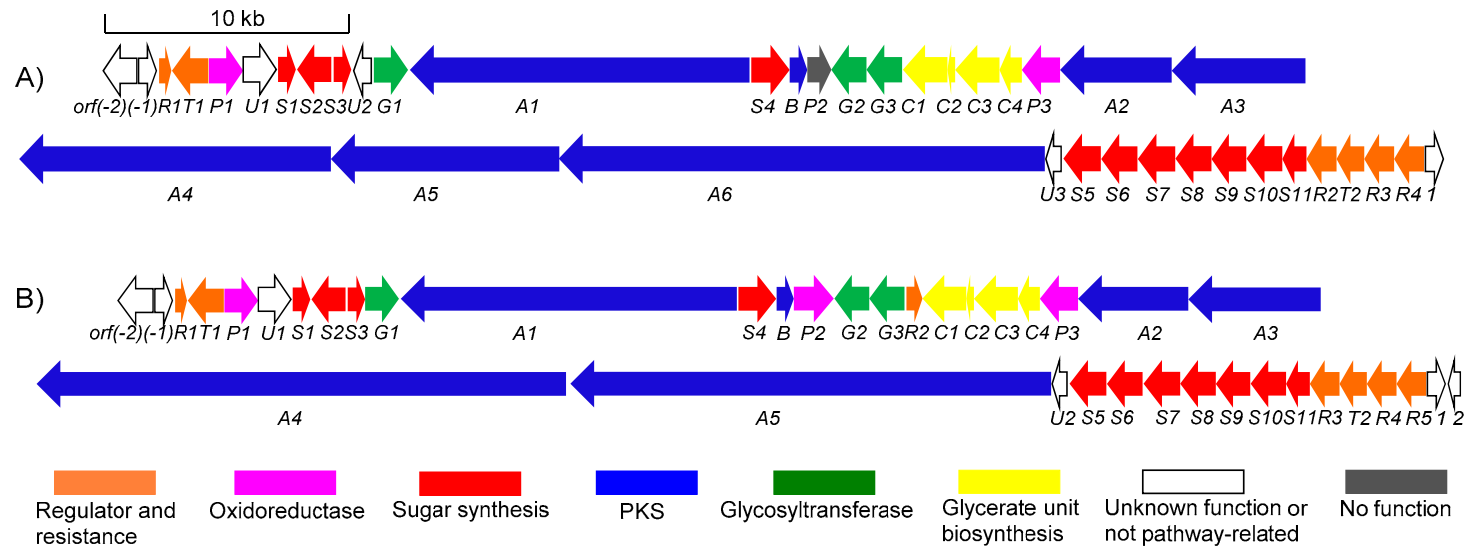

Figure 4. Genetic organizations: (A) the lbp BGC from S. olivaceus SCSIO T05; (B) the lob BGC from S. sp. SCSIO 01127.
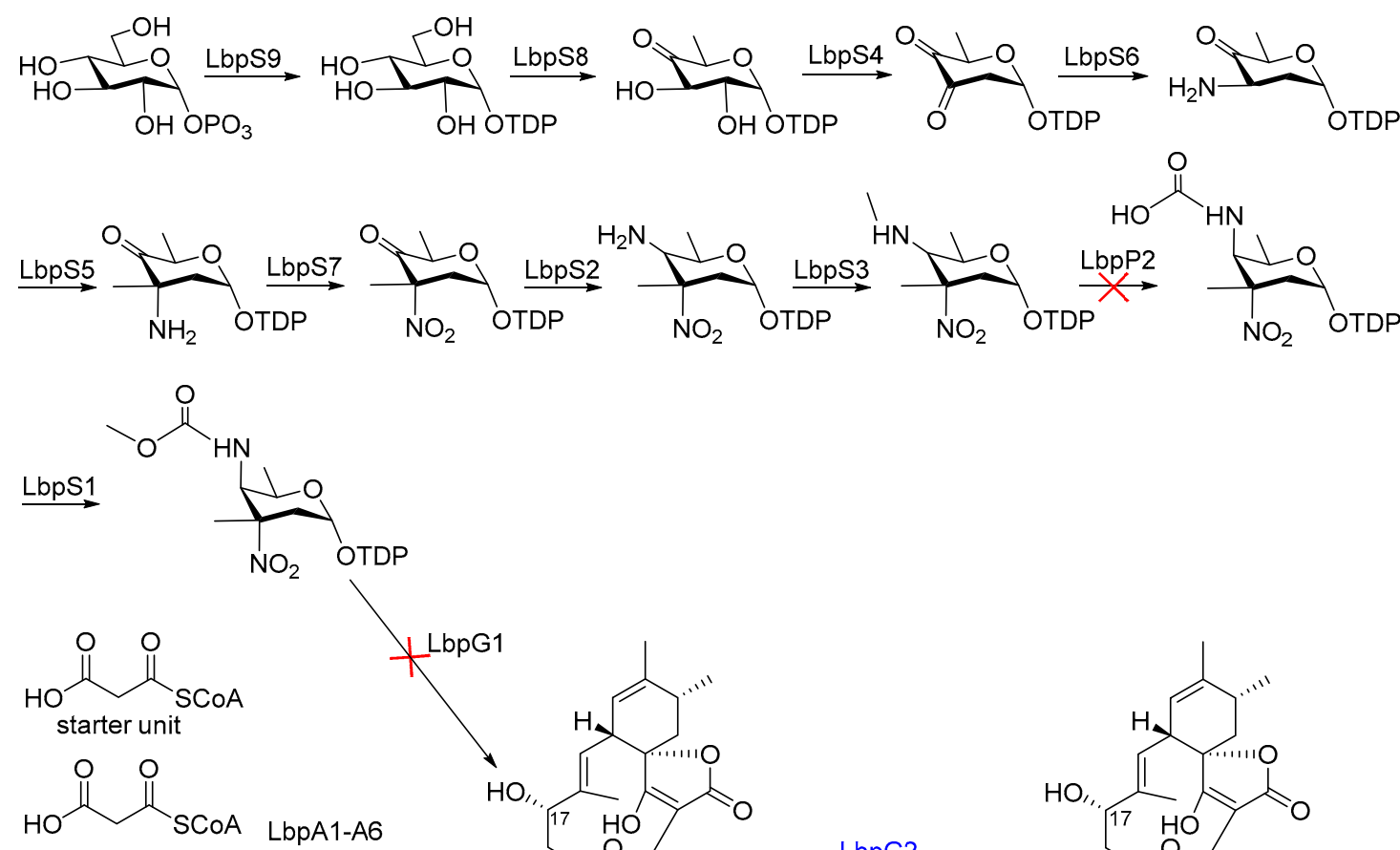

malonyl $\mathrm{CoA}, 5 \times \quad \mathrm{LbpB}, \mathrm{LbpC} 1$

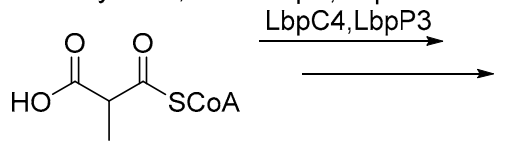

methylmalonyl $\mathrm{CoA}, 6 \times$

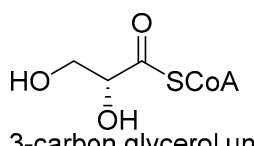

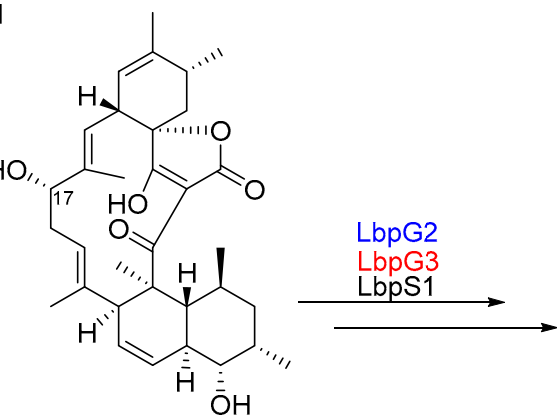

LBP aglycon

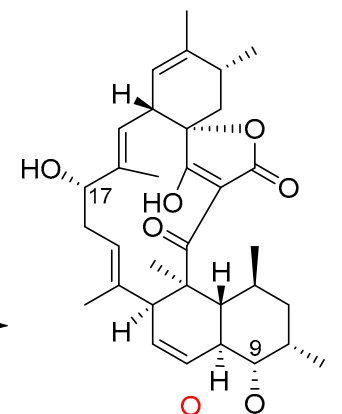

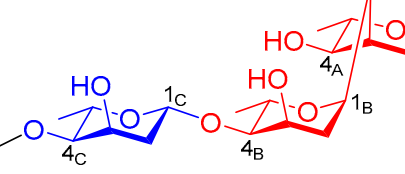

lobophorin CR4 (1)

Figure 5. Proposed biosynthetic pathway of lobophorin CR4. 
Table 3. Deduced function of open reading frames (ORFs) in the $l b p$ BGC.

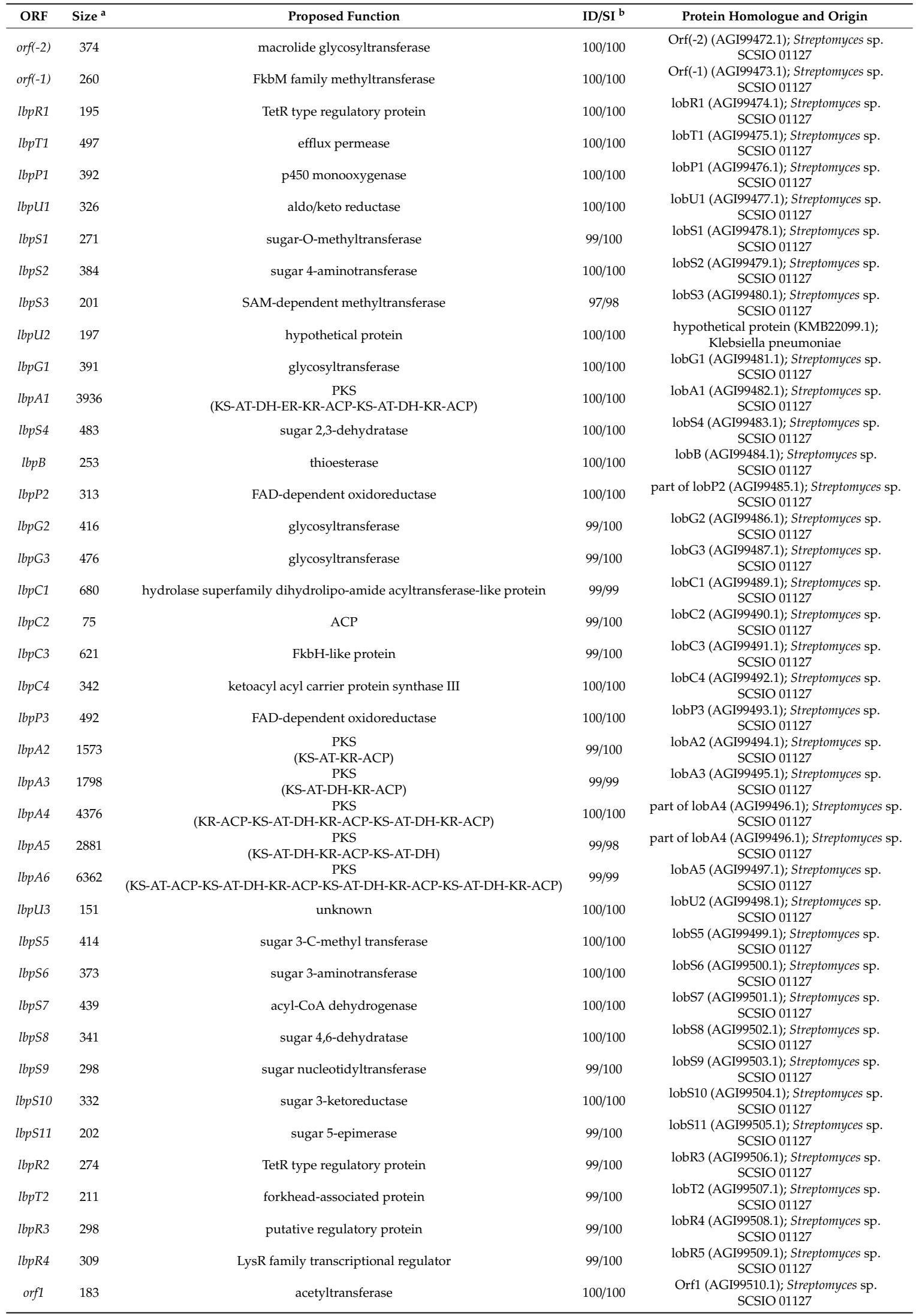

${ }^{\mathrm{a}}$ Amino acids. ${ }^{\mathrm{b}}$ Identity/similarity. 
To demonstrate the validity of the putative $l b p$ BGC, $l b p C 4$ coding for ketosynthase-III-like protein, which incorporates a 3-carbon glycerol unit into the biosynthetic precursor LOB aglycon [11], was disrupted by using PCR-targeting methods. As expected, the production of lobophorin CR4 was completely blocked in S. olivaceus SCSIO T05/ $\Delta r s d K_{2} / \Delta x m c P / \Delta l b p C 4$ (S. olivaceus SCSIO T05RXL) (Figure 2, trace iv), demonstrating that the $l b p$ BGC is indeed responsible for lobophorin biosynthesis. With high similarity to the lob BGC, the $\operatorname{lbp}$ BGC accounts for lobophorin CR4 without the attachment of kijanose to $\mathrm{C} 17-\mathrm{OH}$, rather than lobophorins $\mathrm{A}$ and $\mathrm{B}$ in lob. Based on bioinformatics analysis, a series of enzymes are proposed to be involved in kijanose biosynthesis (Figure 5) [7]. Among them, the amino acid sequence of the putative FAD-dependent oxidoreductase LbpP2 is far shorter than its homologues LobP2 [11] and KijB3 [7]. KijB3 is proposed to oxidize the methyl group to a carboxylate group, essential for the generation of the kijanose moiety [7]. Multiple protein sequence alignments of LbpP2, LobP2, and KijB3 revealed that the conserved FAD binding domain is missing in LbpP2 (Figure S5). Thus, we speculate that LbpP2 is nonfunctional, failing to catalyze the carboxylation and hinder the generation of kijanose.

Given the high similarity of LbpG3 and LobG3, we envision that LbpG3 has a similar function as LobG3, a glycosyltransferase from S. sp. SCSIO 01127, tandemly attaching the first two L-digitoxose at C-9 in lobophorins [11]. LbpG2 has 99\% similarity to LobG2, another glycosyltransferase from the same strain, which was established to transfer the terminal L-digitoxose [11]. Both LbpG2 and LbpG3 are likely to be involved in the transfers of three sugar units, sugars A, B, and C, in lobophorin CR4 (Figure 5), consistent with the metabolite profile of $\triangle l o b G 1$ in S. sp. SCSIO 01127 [11].

\section{Experimental Section}

\subsection{General Experimental Procedures}

The plasmids and bacteria used are listed in Table S1. Streptomyces olivaceus SCSIO T05 and its mutants were incubated on modified ISP-4 medium [25] with 3\% sea salt and fermented in modified RA medium [19]. All cultures for Streptomyces were incubated at $28^{\circ} \mathrm{C}$. Luria-Bertani (LB) medium was used for E. coli, with appropriate antibiotics added at a final concentration of $100 \mu \mathrm{g} / \mathrm{mL}$ of ampicillin (Amp), $50 \mu \mathrm{g} / \mathrm{mL}$ of kanamycin (Kan), $50 \mu \mathrm{g} / \mathrm{mL}$ of apramycin (Apr), $25 \mu \mathrm{g} / \mathrm{mL}$ of chloroamphenicol (Cml), and $50 \mu \mathrm{g} / \mathrm{mL}$ of trimethoprim (TMP).

A 1260 infinity system (Agilent, Santa Clara, CA, USA), which uses a Phenomenex Prodigy ODS (2) column $(150 \times 4.6 \mathrm{~mm}, 5 \mu \mathrm{m}$, USA), was used for HPLC-based analyses. Silica gel with the size of 100-200 mesh (Jiangpeng Silica gel development, Inc., Shandong, China) was used for column chromatography (CC). A Primaide 1110 solvent delivery module, which is equipped with a 1430 photodiode array detector (Hitachi, Tokyo, Japan) and uses a YMC-Pack ODS-A column $(250 \mathrm{~mm}$ $\times 10 \mathrm{~mm}, 5 \mu \mathrm{m}$ ), was used for semi-preparative HPLC. A MaXis Q-TOF mass spectrometer (Bruker, Billerica, MA, USA) was used to acquire high-resolution mass spectral data. An MCP-500 polarimeter (Anton Paar, Graz, Austria) was used to record optical rotations. A Bruker Avance 500 was used to record NMR spectra. Carbon signals and the residual proton signals of DMSO- $d_{6}$ were used for calibration $\left(\delta_{\mathrm{C}} 39.52\right.$ and $\left.\delta_{\mathrm{H}} 2.50\right)$.

\subsection{Genome Sequencing and Bioinformatic Analysis}

Whole genome scanning and annotation of S. olivaceus SCSIO T05 were acquired by the single-molecule real-time (SMRT) sequencing technology (PacBio) at Shanghai Majorbio Bio-Pharm Technology Co., Ltd (Shanghai, China). AntiSMASH (AntiSMASH 5.0, available at http://antismash. secondarymetabolites.org/) was used to analyze and assess the potential BGCs. FramePlot (FramePlot 4.0 beta, available at http://nocardia.nih.go.jp/fp4/) was used to analyze ORFs whose functions were predicted based on an online BLAST program (http://blast.ncbi.nlm.nih.gov/). 


\subsection{Construction of a "Triple-Deletion" Mutant Strain}

Gene $l b p C 4$ from the $l b p$ BGC was inactivated by the REDIRECT protocol [26]. All primers used in this study are listed in Table S2. $L b p C 4$ was replaced by the apramycin resistance gene oriT/aac(3)IV fragment in the target cosmids $01-07 \mathrm{D}$ or 21-02E. The target mutant clones, S. olivaceus SCSIO T05RXL, were accomplished as previously described $[19,23,24]$.

\subsection{Fermentation and HPLC-based Analyses of S. olivaceus SCSIO T05 and Its Mutants}

The Streptomyces used in this study were incubated in modified ISP-4 medium plates for 2-3 $\mathrm{d}$. For fermentation, a portion of mycelium and spores was seeded into $50 \mathrm{~mL}$ of modified RA medium in a $250 \mathrm{~mL}$ flask and then shaken at $200 \mathrm{rpm}$ and $28^{\circ} \mathrm{C}$ for $8 \mathrm{~d}$. The cultures were extracted with an equal volume of butanone. Organic phases were then dissolved in $\mathrm{CH}_{3} \mathrm{OH}(1 \mathrm{~mL})$ after having been evaporated to dryness, and $40 \mu \mathrm{L}$ of each relevant sample was injected for HPLC-based analysis. The UV detection was at $254 \mathrm{~nm}$. Solvent $\mathrm{A}$ is composed of $85 \% \mathrm{ddH}_{2} \mathrm{O}$ and $15 \% \mathrm{CH}_{3} \mathrm{CN}$, supplemented with $0.1 \%$ HOAc. Solvent $\mathrm{B}$ is composed of $85 \% \mathrm{CH}_{3} \mathrm{CN}$ and $15 \% \mathrm{ddH}_{2} \mathrm{O}$, supplemented with $0.1 \%$ HOAc. Samples were analyzed via the following method: a linear gradient from $0 \%$ to $80 \%$ solvent $\mathrm{B}$ in $20 \mathrm{~min}$, and then, from $80 \%$ to $100 \%$ solvent B for $1.5 \mathrm{~min}$, finally eluted with $100 \%$ solvent B in $6.5 \mathrm{~min}$. The flow rate was $1.0 \mathrm{~mL} / \mathrm{min}$.

\subsection{Production, Isolation, and Structure Elucidation of Lobophorin CR4}

The mycelium of S. olivaceus SCSIO T05RX were inoculated into $50 \mathrm{~mL}$ of modified-RA medium and then shaken at $200 \mathrm{rpm}$ and $28^{\circ} \mathrm{C}$ for $2 \mathrm{~d}$, to gain the seed cultures. After that, the seed cultures were transferred into $150 \mathrm{~mL}$ of modified-RA medium and shaken at $200 \mathrm{rpm}$ and $28^{\circ} \mathrm{C}$ for $8 \mathrm{~d}$. After the large-scale fermentation was accomplished, a total of $12 \mathrm{~L}$ of the growth culture was centrifuged at $4000 \mathrm{~g}$ for $10 \mathrm{~min}$ to separate the supernatant and mycelium and further extracted by butanone and acetone, respectively. The two organic phases were concentrated (via solvent removal under vacuum), and the residues were combined. The combined sample was subjected to normal phase silica gel CC eluted with $\mathrm{CHCl}_{3}-\mathrm{CH}_{3} \mathrm{OH}$ (100:0, 98:2, 96:4, 94:6, 92:8, 90:10, 85:15, 80:20, 70:30, 50:50, v/v, each solvent combination in $250 \mathrm{~mL}$ volume) to give ten fractions (AFr.1-AFr.10). Fractions A1-A3 were purified to afford the accumulation of compound $\mathbf{1}(98 \mathrm{mg}$ ), by preparative HPLC, eluting with $90 \%$ solvent $\mathrm{B}$ (A: $\mathrm{H}_{2} \mathrm{O} ; \mathrm{B}: \mathrm{CH}_{3} \mathrm{CN}$ ) over the course of $30 \mathrm{~min}$. The flowrate was $2.5 \mathrm{~mL} / \mathrm{min}$ and the $\mathrm{UV}$ detection was at $254 \mathrm{~nm}$. The purified compound was subjected to MS, ${ }^{1} \mathrm{H}$, and ${ }^{13} \mathrm{C}$ NMR spectra measurements and elucidated as a known intermediate 3 during lobophorins A and B biosynthesis [11], and we named it lobophorin CR4 (1).

\section{Conclusions}

In this study, we acquired the complete genome sequence of S. olivaceus SCSIO T05. The biosynthetically talented strain harbors 37 putative BGCs analyzed by antiSMASH. To explore the biosynthetic potential of this strain, metabolic engineering and genome mining were performed. The major anthracenes and indolosesquiterpenes biosynthetic pathways were blocked, and an orphan spirotetronate antibiotics BGC $(l b p)$ was activated in S. olivaceus SCSIO T05, leading to the isolation and identification of one known compound, lobophorin CR4. We have identified the $l b p$ BGC accounting for lobophorin biosynthesis by gene-disruption experiments and bioinformatics analysis. The production of lobophorin CR4 without the attachment of D-kijanose to $\mathrm{C} 17-\mathrm{OH}$ was on account that the nonfunctional FAD-dependent oxidoreductase LbpP2 failed to generate D-kijanose. This work highlights that metabolic engineering and genome mining are the effective ways to turn on putative orphan or silent BGCs to acquire new NPs for drugs discovery.

Supplementary Materials: The following are available online at http://www.mdpi.com/1660-3397/17/10/593/s1. This section includes HRESIMS, 1D NMR spectra for compound 1, construction of $\triangle l b p C 4$. 
Author Contributions: C.Z. performed the experiments and wrote the draft manuscript. W.D. performed the isolation of compound 1. X.Q. helped to perform the sequence alignments. J.J. supervised the whole work and edited the manuscript. All authors read and approved the final manuscript.

Funding: This research was funded by the National Natural Science Foundation of China (81425022, U1706206, and U1501223), and Natural Science Foundation of Guangdong Province (2016A030312014).

Acknowledgments: We are grateful to Aijun Sun, Xiaohong Zheng, Yun Zhang, Xuan Ma, and Zhihui Xiao, in the analytical facility center of the SCSIO for recording MS and NMR data.

Conflicts of Interest: The authors declare no conflicts of interest.

\section{References}

1. Berdy, J. Bioactive microbial metabolites. J. Antibiot. 2005, 58, 1-26. [CrossRef] [PubMed]

2. Carroll, A.R.; Copp, B.R.; Davis, R.A.; Keyzers, R.A.; Prinsep, M.R. Marine natural products. Nat. Prod. Rep. 2019, 36, 122-173. [CrossRef] [PubMed]

3. Saha, S.; Zhang, W.; Zhang, G.; Zhu, Y.; Chen, Y.; Liu, W.; Yuan, C.; Zhang, Q.; Zhang, H.; Zhang, L.; et al. Activation and characterization of a cryptic gene cluster reveals a cyclization cascade for polycyclic tetramate macrolactams. Chem. Sci. 2017, 8, 1607-1612. [CrossRef] [PubMed]

4. Chen, R.; Zhang, Q.; Tan, B.; Zheng, L.; Li, H.; Zhu, Y.; Zhang, C. Genome Mining and Activation of a Silent PKS/NRPS Gene Cluster Direct the Production of Totopotensamides. Org. Lett. 2017, 19, 5697-5700. [CrossRef]

5. Li, Y.; Zhang, C.; Liu, C.; Ju, J.; Ma, J. Genome sequencing of Streptomyces atratus SCSIO ZH16 and activation production of nocardamine via metabolic engineering. Front. Microbiol. 2018, 9, 1269. [CrossRef]

6. Sun, C.; Yang, Z.; Zhang, C.; Liu, Z.; He, J.; Liu, Q.; Zhang, T.; Ju, J.; Ma, J. Genome Mining of Streptomyces atratus SCSIO ZH16: Discovery of Atratumycin and Identification of Its Biosynthetic Gene Cluster. Org. Lett. 2019, 21, 1453-1457. [CrossRef]

7. Zhang, H.; White-Phillip, J.A.; Melançon, C.E.; Kwon, H.J.; Yu, W.L.; Liu, H.W. Elucidation of the Kijanimicin Gene Cluster: Insights into the Biosynthesis of Spirotetronate Antibiotics and Nitrosugars. J. Am. Chem. Soc. 2007, 129, 14670-14683. [CrossRef]

8. Fang, J.; Zhang, Y.; Huang, L.; Jia, X.; Zhang, Q.; Zhang, X.; Tang, G.; Liu, W. Cloning and Characterization of the Tetrocarcin A Gene Cluster from Micromonospora chalcea NRRL 11289 Reveals a Highly Conserved Strategy for Tetronate Biosynthesis in Spirotetronate Antibiotics. J. Bacteriol. 2008, 190, 6014-6025. [CrossRef]

9. Wei, R.B.; Xi, T.; Li, J.; Wang, P.; Li, F.C.; Lin, Y.C.; Qin, S. Lobophorin C and D, New Kijanimicin Derivatives from a Marine Sponge-Associated Actinomycetal Strain AZS17. Mar. Drugs 2011, 9, 359-368. [CrossRef]

10. Niu, S.; Li, S.; Chen, Y.; Tian, X.; Zhang, H.; Zhang, G.; Zhang, W.; Yang, X.; Zhang, S.; Ju, J.; et al. Lobophorins E and F, new spirotetronate antibiotics from a South China Sea-derived Streptomyces sp. SCSIO 01127. J. Antibiolt. 2011, 64, 711. [CrossRef]

11. Li, S.; Xiao, J.; Zhu, Y.; Zhang, G.; Yang, C.; Zhang, H.; Ma, L.; Zhang, C. Dissecting Glycosylation Steps in Lobophorin Biosynthesis Implies an Iterative Glycosyltransferase. Org. Lett. 2013, 15, 1374-1377. [CrossRef] [PubMed]

12. Pan, H.Q.; Zhang, S.Y.; Wang, N.; Li, Z.L.; Hua, H.M.; Hu, J.C.; Wang, S.J. New Spirotetronate Antibiotics, Lobophorins H and I, from a South China Sea-Derived Streptomyces sp. 12A35. Mar. Drugs 2013, 11, 3891-3901. [CrossRef] [PubMed]

13. Chen, C.; Wang, J.; Guo, H.; Hou, W.; Yang, N.; Ren, B.; Liu, M.; Dai, H.; Liu, X.; Song, F.; et al. Three antimycobacterial metabolites identified from a marine-derived Streptomyces sp. MS100061. Appl. Microbiol. Biotechnol. 2013, 97, 3885-3892. [CrossRef] [PubMed]

14. Cruz, P.G.; Fribley, A.M.; Miller, J.R.; Larsen, M.J.; Schultz, P.J.; Jacob, R.T.; Tamayo-Castillo, G.; Kaufman, R.J.; Sherman, D.H. Novel Lobophorins Inhibit Oral Cancer Cell Growth and Induce Atf4- and Chop-Dependent Cell Death in Murine Fibroblasts. ACS Med. Chem. Lett. 2015, 6, 877-881. [CrossRef] [PubMed]

15. Song, C.; Pan, H.; Hu, J. Isolation and identification of a new antibiotic, lobophorin J, from a deep sea-derived Streptomyces sp. 12A35. Chin. J. Antibiot. 2015, 40, 721-727.

16. Yue, C.; Niu, J.; Liu, N.; Lü, Y.; Liu, M.; Li, Y. Cloning and identification of the lobophorin biosynthetic gene cluster from marine Streptomyces olivaceus strain FXJ7.023. Pak. J. Pharm. Sci. 2016, 29, 287-293. [PubMed] 
17. Braña, A.; Sarmiento-Vizcaíno, A.; Osset, M.; Pérez-Victoria, I.; Martín, J.; de Pedro, N.; de la Cruz, M.; Díaz, C.; Vicente, F.; Reyes, F.; et al. Lobophorin K, a new natural product with cytotoxic activity produced by Streptomyces sp. M-207 associated with the deep-sea coral Lophelia pertusa. Mar. Drugs. 2017, 15, 144. [CrossRef]

18. Low, Z.J.; Pang, L.M.; Ding, Y.; Cheang, Q.W.; Hoang, K.L.M.; Tran, H.T.; Li, J.; Liu, X.-W.; Kanagasundaram, Y.; Yang, L.; et al. Identification of a biosynthetic gene cluster for the polyene macrolactam sceliphrolactam in a Streptomyces strain isolated from mangrove sediment. Sci. Rep. 2018, 8, 1594. [CrossRef]

19. Zhang, C.; Sun, C.; Huang, H.; Gui, C.; Wang, L.; Li, Q.; Ju, J. Biosynthetic Baeyer-Villiger Chemistry Enables Access to Two Anthracene Scaffolds from a Single Gene Cluster in Deep-Sea-Derived Streptomyces olivaceus SCSIO T05. J. Nat. Prod. 2018, 81, 1570-1577. [CrossRef]

20. Chin, C.-S.; Alexander, D.H.; Marks, P.; Klammer, A.A.; Drake, J.; Heiner, C.; Clum, A.; Copeland, A.; Huddleston, J.; Eichler, E.E.; et al. Nonhybrid, finished microbial genome assemblies from long-read SMRT sequencing data. Nat. Methods 2013, 10, 563-569. [CrossRef]

21. Blin, K.; Shaw, S.; Steinke, K.; Villebro, R.; Ziemert, N.; Lee, S.Y.; Medema, M.H.; Weber, T. AntiSMASH 5.0: Updates to the secondary metabolite genome mining pipeline. Nucleic Acids Res. 2019, 47, W81-W87. [CrossRef] [PubMed]

22. Scherlach, K.; Hertweck, C. Triggering cryptic natural product biosynthesis in microorganisms. Org. Biomol. Chem. 2009, 7, 1753. [CrossRef] [PubMed]

23. Sun, C.; Zhang, C.; Qin, X.; Wei, X.; Liu, Q.; Li, Q.; Ju, J. Genome mining of Streptomyces olivaceus SCSIO T05: Discovery of olimycins A and B and assignment of absolute configurations. Tetrahedron 2018, 74, 199-203. [CrossRef]

24. Zhang, C.; Yang, Z.; Qin, X.; Ma, J.; Sun, C.; Huang, H.; Li, Q.; Ju, J. Genome Mining for Mycemycin: Discovery and Elucidation of Related Methylation and Chlorination Biosynthetic Chemistries. Org. Lett. 2018, 20, 7633-7636. [CrossRef] [PubMed]

25. Liu, W.; Shen, B. Genes for Production of the Enediyne Antitumor Antibiotic C-1027 in Streptomyces globisporus Are Clustered with the cagA Gene That Encodes the C-1027 Apoprotein. Antimicrob. Agents Chemother. 2000, 44, 382-392. [CrossRef] [PubMed]

26. Gust, B.; Challis, G.L.; Fowler, K.; Kieser, T.; Chater, K.F. PCR-targeted Streptomyces gene replacement identifies a protein domain needed for biosynthesis of the sesquiterpene soil odor geosmin. Proc. Natl. Acad. Sci. USA 2003, 100, 1541-1546. [CrossRef] [PubMed] 